\title{
Finite Element Modelling of Different 2D Re- Entrant Structures of Auxetic Materials
}

\author{
[Mozafar Shokri Rad, Zaini Ahmad*]
}

\begin{abstract}
Auxetic materials exhibit a unique characteristics when subjected to uniaxial loading. Various structures have been used to model these materials. Among most important auxetic structures, re-entrant structures are of interest in this present study. These structures have different shapes in which are known as lozenge grids, sinusoidal ligaments, square grids, double arrowhead, and structurally hexagonal re-entrant honeycomb could be named. In this paper, finite element approach for the abovementioned structures was employed to obtain basic mechanical properties including Poisson's ratio and elastic modulus. The study aims at investigating the effect of cross sectional geometry on mechanical properties. For each structure, three different cross sectional geometries were numerically examined. It is evident that mechanical properties of the material could be controlled by changing the geometry of the cross section. The primary outcome of the study is the design guideline on the effect of cross sectional geometry on mechanical properties of auxetic structures.
\end{abstract}

Keywords - auxetic; finite element; cross section; re-entrant, Poisson's ratio, elastic modulus

\section{Introduction}

For the past decades, different geometrical structures exhibiting auxetic behaviour have been fabricated and tested to obtain their mechanical properties. Undoubtedly, these structures are useful since they assist many researchers to comprehend on how auxetic behaviour is obtained and how auxetic materials can be fabricated. Computational procedure is still sparse in predicting their mechanical properties.

Among the most important classes of such auxetic structures are re-entrant structures in which have been investigated by some researchers [1-6]. The other classes are namely chiral structures [7-9], rotating rigid/semi-rigid units[10-14], angle-ply laminates [15, 16], hard molecules [17-20], micro porous polymers [21-23], and liquid crystalline polymer [24 -26]. In particular, the important 2D re-entrant structures are structurally hexagonal honeycomb, double arrow head structures, structures formed from lozenge grids, structure formed from sinusoidal ligaments, and structure formed from square grids [27]. Man-made auxetic materials were first designed by Theocaris et al. [28]. In this study, the design of material was based on structure of arrays with polygonal-shaped inclusions and reentrant corners.

Zaini Ahmad

Universiti Teknologi Malaysia

Malaysia

Mozafar Shokri Rad

Universiti Teknologi Malaysia

Malaysia
In the present study, finite element technique has been employed in modelling these structures to represent the characteristic of the auxetic re-entrant structures. Five different shapes of 2D re-entrant structures have been considered to examine the influence of cross sectional geometry on the mechanical properties. It is evident that mechanical properties of auxetic material are controllable, thus facilitating the fabrication technique used in preparing samples in the laboratory.

\section{Finite element modeling of different $2 D$ re-entrant structures of auxetic materials}

There are different re-entrant structures introduced by Liu et al [27]. Based on their shape, they were named lozenge grids, sinusoidal ligaments, square grids, double arrowhead, and structurally hexagonal re-entrant honeycomb. These structures are shown in Figure 1.

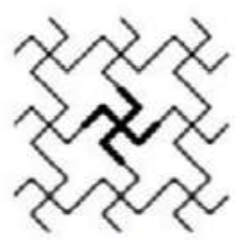

(a)

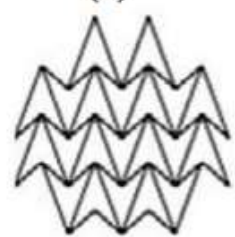

(d)



(b)

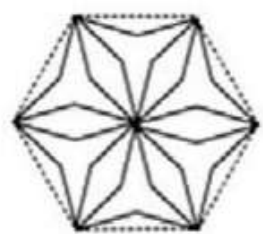

(e)

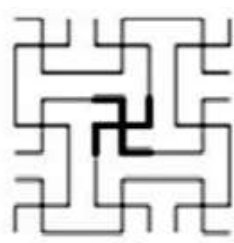

(c)
Figure 1. Different shapes of 2D re-entrant structures. (a): Lozenge grids. (b): Sinusoidal ligaments. (c): Square grids. (d): Double arrowhead. (e): Structurally hexagonal re-entrant honeycomb [27]

In the present paper, finite element modelling of the abovementioned structures has been carried out to obtain their basic mechanical properties in terms of Poisson's ratio and elasticity modulus. Element used in the modelling is linear tetrahedral, type C3D4. The optimized mesh size was determined from the convergent study. Each model was loaded along $x$ direction with the imposed normal deflections. The Poisson's ratio and Young's modulus were then calculated.

Using the defined values for the lengths of connecting rods and their rigid property, one can determine strains $\varepsilon_{x}, \varepsilon_{y}$ and Poisson's ratio $v_{x y}$ of the $2 \mathrm{D}$ re-entrant structures shown in Figure 1 parametrically by using the following equations. 
Proc. of the Second Intl. Conf. on Advances in Mechanical and Automation Engineering - MAE 2015 Copyright (C) Institute of Research Engineers and Doctors, USA .All rights reserved.

ISBN: 978-1-63248-045-3 doi: 10.15224/ 978-1-63248-045-3-38

$$
\begin{aligned}
& \varepsilon_{x}=\frac{\Delta L}{L}, \varepsilon_{y}=\frac{\Delta H}{H} \\
& v_{x y}=-\frac{\varepsilon_{y}}{\varepsilon_{x}}=-\frac{(\Delta H)(L)}{(\Delta L)(H)}
\end{aligned}
$$

Where $H$ and $L$ are height and width of the 2D structure, respectively.

Normal stress along $x, \sigma_{1}$, is defined as the whole external force applied to the structure divided by the area perpendicular to the loading direction at the origion. This can be expressed as:

$$
\sigma_{1}=\frac{F}{A_{1}}=\frac{F}{(H t)}
$$

Where $t$ is the thickness of the structure.

As a result, one can determine elastic modulus of the $2 \mathrm{D}$ structure parametrically:

$$
E_{1}=\frac{\sigma_{1}}{\varepsilon_{1}}=\frac{\frac{F}{(H t)}}{\frac{\Delta L}{L}}
$$

\section{Results and discussion}

As abovementioned, finite element for each structure has been developed. For each model, three different cross sections were taken into consideration. These cross sections are square, circle, and triangle in which cross section areas for each model were held fixed. The displacement contours obtained from finite element modelling is shown in Figures 2 and 3.

TABLE I. FINITE ELEMENT RESULTS OF POISON'S RATIO AND ELASTIC MODULUS.

\begin{tabular}{|c|c|c|c|c|c|}
\hline $\begin{array}{c}\text { Model } \\
\text { No }\end{array}$ & $\begin{array}{c}\text { Cross } \\
\text { Section }\end{array}$ & $\varepsilon_{x}$ & $\varepsilon_{y}$ & $v_{x y}=-\frac{\varepsilon_{x}}{\varepsilon_{x}}$ & $\mathrm{E}_{1}=\frac{\sigma_{1}}{\varepsilon_{1}}$ \\
\hline \multirow{4}{*}{1} & Square & 0.1127 & 0.0292 & -0.2591 & $1051.5 \mathrm{MPa}$ \\
\cline { 2 - 6 } & Circle & 0.1127 & 0.0291 & -0.2472 & $1480 \mathrm{MPa}$ \\
\cline { 2 - 6 } & Triangle & 0.1127 & 0.0278 & -0.2378 & $2617 \mathrm{MPa}$ \\
\hline \multirow{4}{*}{2} & Square & 0.0556 & 0.0387 & -0.696 & $2131.1 \mathrm{MPa}$ \\
\cline { 2 - 6 } & Circle & 0.0556 & 0.0345 & -0.621 & $2580.3 \mathrm{MPa}$ \\
\cline { 2 - 6 } & Triangle & 0.0556 & 0.0355 & -0.639 & $3124.5 \mathrm{MPa}$ \\
\hline \multirow{3}{*}{3} & Square & 0.0478 & 0.02 & -0.421 & $2832.5 \mathrm{MPa}$ \\
\cline { 2 - 6 } & Circle & 0.0478 & 0.0187 & -0.392 & $3721.2 \mathrm{MPa}$ \\
\cline { 2 - 6 } & Triangle & 0.0478 & 0.0148 & -0.311 & $4239.6 \mathrm{MPa}$ \\
\hline \multirow{3}{*}{4} & Square & 0.0232 & 0.0283 & -1.2241 & $4020 \mathrm{MPa}$ \\
\cline { 2 - 6 } & Circle & 0.0232 & 0.0258 & -1.1124 & $6010 \mathrm{MPa}$ \\
\cline { 2 - 6 } & Triangle & 0.0232 & 0.0214 & -0.925 & $8040 \mathrm{MPa}$ \\
\hline \multirow{3}{*}{5} & Square & 0.025 & 0.0292 & -1.168 & $3608.4 \mathrm{MPa}$ \\
\cline { 2 - 6 } & Circle & 0.025 & 0.0268 & -1.0721 & $4728.4 \mathrm{MPa}$ \\
\cline { 2 - 6 } & Triangle & 0.025 & 0.022 & -0.885 & $6121.2 \mathrm{MPa}$ \\
\hline \multirow{3}{*}{} & & & & & \\
\hline \multirow{3}{*}{} & & & & & \\
\hline
\end{tabular}

Table 1 shows the results of finite element simulation in which the first three models, second three models, third three models, fourth three models and last three models are lozenge grids structures, sinusoidal ligaments, square grids, double arrowhead, hexagonal re-entrant honeycomb structures, respectively.
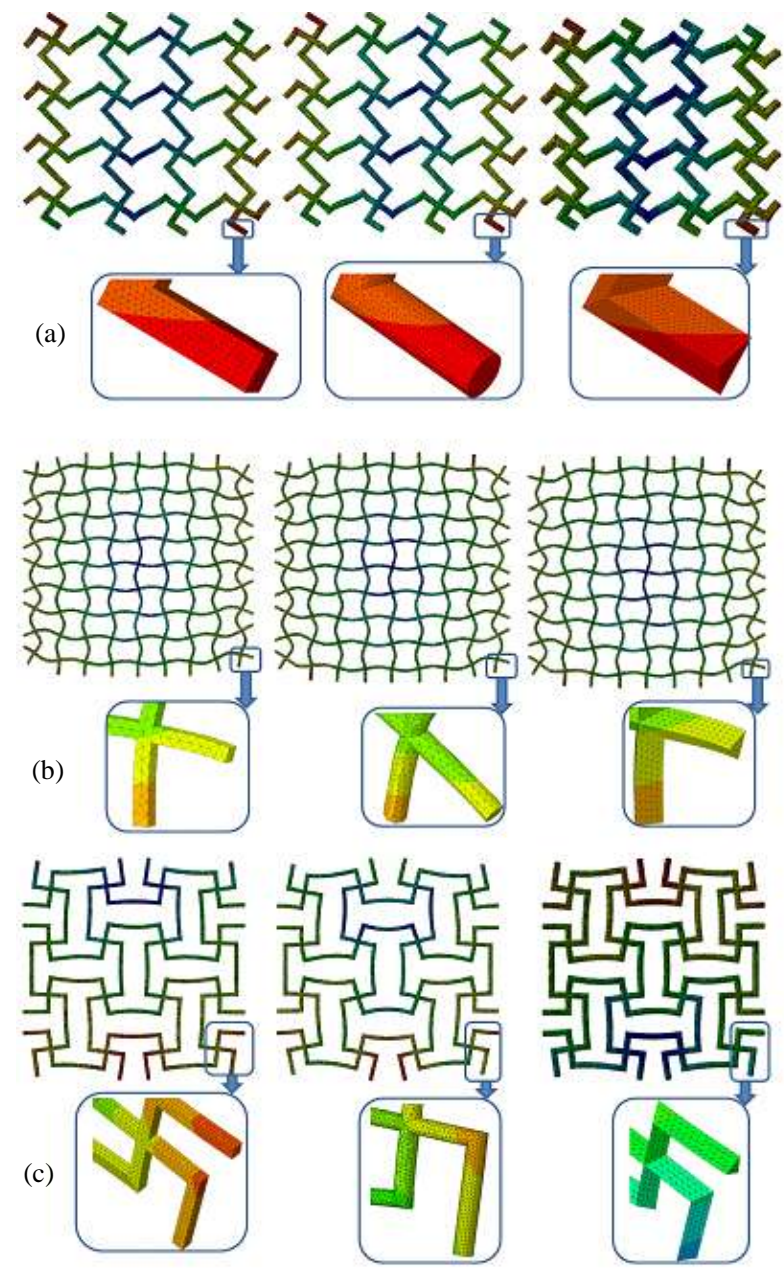

Figure 2. Finite element models of 2D re-entrant structures. (a): lozenge grids. (b): sinusoidal ligaments. (c): square grids.

Overall, the results show that the shape of cross section has a great influence on the mechanical properties. In terms of Poisson's ratio, the auxeticity varies with the geometrical shapes of the cross section. It is noteworthy that the square cross section exhibits the highest auxeticity behaviour compared with the other two cross sections. Furthermore, the elastic modulus of triangular cross sections is much more higher than that of other two cross sections as evidenced in Table 1. As a guideline, if the higher stiffness of the auxetic re-entrant structure is needed, the triangular crossectional shapes may be employed in simulating auxeticity of any model. 


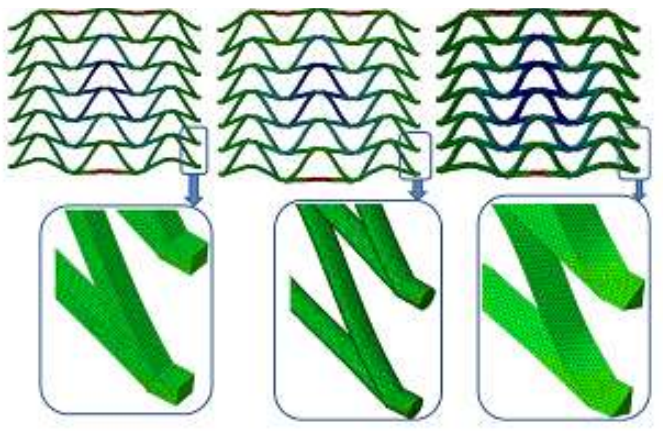

(a)

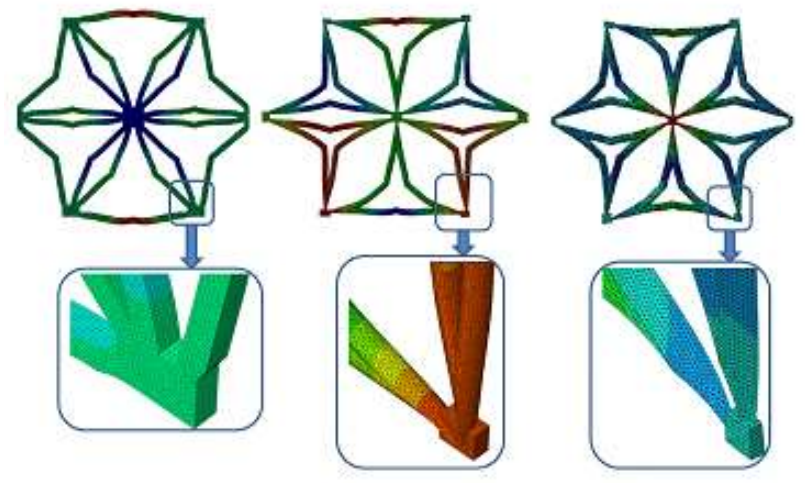

(b)

Figure 3. Finite element models of 2D re-entrant structures. (a): double arrowhead. (b): structurally hexagonal re-entrant honeycomb.

\section{Conclusion}

Different configurations for 2D re-entrant structures of auxetic materials from previous studies have been developed using finite element technique to examine their mechanical properties. The effect of cross section geometry on Poisson's ratio and elastic modulus has then been investigated numerically. For each structure, three different cross sectional geometries with an identical cross-sectional area were considered. The results show that the shape of cross section has a great influence on the mechanical properties. It is discovered that the elastic modulus for triangular cross sections is greater than that for the other two cross sections. Thereby, manipulation of stiffness properties including Poisson's ratio as well as fracture toughness can be performed by varying the cellular structural parameters. This research provides a basis for experimental additive manufacturing processes such as electron beam melting or selective laser sintering.

\section{Acknowledgment}

This project is funded by the Ministry of Education (MOE), Government of Malaysia through Universiti Teknologi Malaysia research grants: R.J130000.7824.4L105 and Fundamental Research Grant Scheme (FRGS) Vote No:R.J 130000.7824.4F248. Sincere acknowledgement also go to Universiti Teknologi Malaysia (UTM) for the continous support in completing this project.

\section{References}

[1] Lee J, Choi JB, Choi K, Application of homogenization FEM analysis to regular and re-entrant honeycomb structures. J. Mater.Sci., 1996, 31: 4105-4110.

[2] Gaspar N, Ren XJ, Smith CW, Grima JN, Evans KE, Honeycombs with Auxetic Behaviour. Acta. Mater., 2005, 53 (8): 2439-2445.

[3] Gibson LJ, Ashby MF, Schajer GS, Robertson CI, The Mechanics of Two Dimensional Cellular Materials. Proc Lond. Royal Soc, 1982, 382: 25-42.

[4] Theocaris PS, Stavroulakis, The homogenization method for the study of variation of Poisson's ratio in fiber composites. Arch. Appl. Mech., 1998, 68: 281-295.

[5] Lim TC, Constitutive relationship of a material with unconventional Poisson's ratio. J. Mater. Sci. Lett., 2003, 22: 1783-1786.

[6] Li Y, The anisotropic behaviour of Poisson's ratio, Young's modulus and shear modulus in hexagonal materials. Phys. Stat. Soil., 1976, 38: $171-175$

[7] Grima JN, Gatt R, Alderson A, Evans KE, On the potential of connected stars as auxetic systems. Mol. Simul., 2005, 31: 925-935.

[8] Grima JN, Gatt R, Farrugia PS, On the properties of auxeticmetatetrachiral structures. Phys. Stat. Sol., 2008, 245: 511-520

[9] Prall D, Lakes RS, Properties of a chiral honeycomb with a poisson's ratio of -1. Int. J. Mech. Sci, 1997, 39: 305-314.

[10] Spadoni A, Ruzzene M, Scarpa F, Global and local linear buckling behaviour of a chiral cellular structure. Phys. Stat. Sol, 2005, 242:695709.

[11] Bornengo D, Scarpa F, Remillat C, Evaluation of hexagonal chiral structure for morphing airfoil concept.Proc IME GJ Aero Eng., 2005, 219: 185-192.

[12] Ishibashi Y, Iwata MJ, A Microscopic Model of a Negative Poisson's Ratio in Some Crystals. J. Phys. Soc, Jpn., 2000, 69: 2702-2703.

[13] Vasiliev AA, Dimitriev SV, Ishibashi Y, Shinegari T, Phys. Rev., 2002, 65: 094101.

[14] Grima JN, Gatt R, Ravirala N, Alderson A, Evans KE, Negative Poisson's ratios in cellular foam materials. Mat. Sci. Eng., 2006, 423: 214-218.

[15] Grima JN, Gatt R, Alderson A, Evans KE, On the Auxetic Properties of 'Rotating Rectangles' with Different Connectivity Jpn. J. Phys. Soc., 2005, 74: 2866-2867.

[16] Milton GW, Composite materials with Poisson's ratios close to -1.J. Mech. Phys. Solids, 1992, 40: 1105-1137.

[17] Hine PJ, Duckett RA, Ward IM, Negative Poisson's ratios inangle-ply laminates. J. Mater. Sci. Lett., 1997, 16: 541-544.

[18] Wojciechowski KW, Constant thermodynamic tension Monte Carlo studies of elastic properties of a two-dimensional system of hard cyclic hexamers. Mol. Phys., 1987, 61: 1247-1258.

[19] Wojciechowski KW, Remarks on "Poisson Ratio beyond the Limits of the Elasticity Theory". J. Phys. Soc. Jpn., 2003, 72: 1819-1820.

[20] Wojciechowski KW, Non-chiral, molecular model of negative Poisson ratio in two dimensions. J. Phys. A: Math. Gen., 2003, 36: 11765-11778.

[21] Tretiakov KV, Wojciechowski KW, Poisson's ratio of simple planar isotropic solids in two dimensions. Phys. Stat. Sol, 2007, 244: 10381046.

[22] Caddock BD, Evans KE, Microporous materials with negative Poisson's ratios. I. Microstructure and mechanical properties. J. Phys. D: Appl. Phys., 1989, 22: 1877-1882.

[23] Alderson A, Alderson KL, Auxetic materials. Proc IME G.J. Aero.Eng., 2007, 221: 565-575.

[24] Alderson KL, Alderson A, Evans KE, The interpretation of the straindependent Poisson's ratio in auxetic polyethylene. J. Strain Anal. Eng. Design, 1997, 32: 201-212.

[25] He CB, Liu PW, Mc Mullan PJ, Griffin AC, Toward molecular auxetics: Main chain liquid crystalline polymers consisting of laterally attached para-quaterphenyls. Phys. Stat. Sol., 2005, 242: 576-584.

[26] Aldred P, Moratti SC, Dynamic simulations of potentially auxetic liquid-crystalline polymers incorporating swivelling mesogens. Mol.Simul., 2005, 31: 883-887. 
[27] Liu $\mathrm{Y}$ and $\mathrm{Hu} \mathrm{H}$, A review on auxetic structures and polymeric materials. Scientific Research and Essays, 2010, Vol. 5 (10), pp. 1052-1063.

[28] P. S. Theocaris, G. E. Stavroulakis, P. D. Panagiotopoulos, Negative Poisson ratios in composites with star-shaped inclusions: a numerical homogenization approach. Archive of applied mechanics., 1997, 67: 274-286.

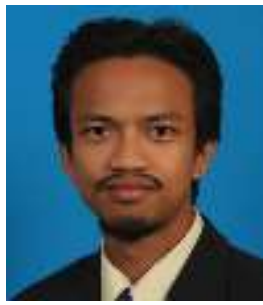

Zaini Ahmad (CEng MIMechE) is currently a senior lecturer in Universiti Teknologi Malaysia. He received his Master of Science (MSc. in Structures, Crashworthiness\& Impact) from Cranfield University, United Kingdom in 2004. He had also obtained his PhD in Structural Impact at Queensland University of Technology, Australia in 2009. Computational solid mechanics is of his primary research interest. Other areas of expertise are finite element modeling, crashworthiness, impact mechanics, crash analysis, thin-walled structures and failure of structure under dynamic loading. He has continuously published a number of refereed scientific publications with Elsevier, Springer, Scientific, etc. He received his professional qualification as

Charted Engineer from the Engineering Council, United Kingdom in 2015 and he is also a member of IAENG, since 2010 .

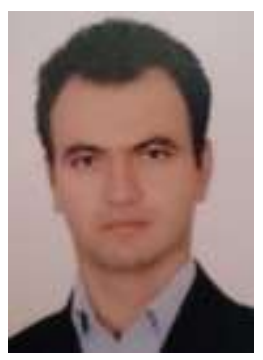

Mozafar Shokri Rad received his BEng and MEng from Iran. He is currently working in the Faculty of Mechanical Engineering, Universiti Teknologi Malaysia, Malaysia as a doctorate student. His research interests are computational solid mechanics, advanced materials, non-linear finite element analysis as well as impact mechanics. He has published several scientific publications with Elsevier, Hindawi, Scientific, etc. 